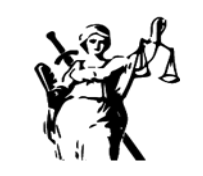

JUSTICIA

ISSN impreso 0124-7441
ISSN digital 2590-4566

\title{
Iniciativas locales: herramienta de mediación para la construcción de paz, Ovejas - Sucre*
}

\author{
Local initiatives: mediation tool for peacebuilding, \\ Ovejas - Sucres \\ Iniciativas locais: ferramenta de mediação para construção \\ da paz, Ovejas - Sucre
}

\author{
(iD) \\ Santander José De La Ossa Guerra \\ Universidad de Sucre, Colombia \\ santander.delaossa@unisucre.edu.co
}

\author{
(iD) \\ Geanny Karin Rendón Blanco \\ Universidad de Caldas, Colombia \\ gea.rendon@gmail.com
}

Recibido: 19 de marzo de 2021 / Aceptado: 28 de marzo de 2021 https://doi.org/10.17081/just.26.39.4723

\begin{abstract}
Resumen
El objetivo de esta investigación fue analizar las iniciativas locales que actúan como herramientas de mediación para la construcción de paz en el municipio de Ovejas, Departamento de Sucre. El método utilizado se enmarcó en el enfoque cualitativo con diseño descriptivo- transversal. La población objeto de investigación, fueron las organizaciones formales o, de hecho, que se han desarrollado en las zonas urbana y rural del municipio de Ovejas, en el Departamento de Sucre como iniciativas locales de paz. Para la recolección de datos, se utilizaron las técnicas de revisión documental y la entrevista abierta. La información se recabó el segundo semestre de 2019. Como resultados, se pudo determinar que, en el municipio de Ovejas se encuentran alrededor de 60 iniciativas locales, representadas en organizaciones campesinas, productivas, de mujeres, de víctimas y parcialidades indígenas; las cuales, han participado de la construcción del PDET, la gestión de reparación colectiva de víctimas del conflicto armado, construcción de políticas públicas, recuperación de la memoria histórica, impulso a la zona de reserva campesina, defensa del territorio y los recursos naturales e impulso a la economía local. Que estas iniciativas son producto de estrategias nacionales y regionales que, con apoyo de organismos internacionales, se han implementado en la región de los Montes de María.
\end{abstract}

PALABRAS CLAVE: Construcción de paz, iniciativas locales, herramientas de mediación, paz territorial. 


\begin{abstract}
The objective of this research was to analyze local initiatives that act as mediation tools for peacebuilding in the municipality of Ovejas, Department of Sucre. The method used was framed in the qualitative approach with a descriptive-cross-sectional design. The population under investigation were formal organizations or, in fact, those that have developed in urban and rural areas of the municipality of Ovejas, in the Department of Sucre as local peace initiatives. For data collection, document review techniques and open interviews were used. The information was collected in the second half of 2019. As a result, it was determined that, in the municipality of Ovejas, there are around 60 local initiatives, represented in peasant organizations, productive, women, victims and indigenous partialities; which have participated in the construction of the PDET, the management of collective reparation for victims of the armed conflict, construction of public policies, recovery of historical memory, promotion of the peasant reserve area, defense of the territory and natural resources and promotion of the local economy. That these initiatives are the product of national and regional strategies that, with the support of international organizations, have been implemented in the Montes de María region.
\end{abstract}

KEYWORDS: Peace building, local initiatives, mediation tools, territorial peace.

Como Citar: De La Ossa Guerra, S. J., \& Rendón Blanco, G. K. (2021). Iniciativas locales: herramienta de mediación para la construcción de paz, Ovejas - Sucre. Justicia, 26(39), 213-224. https://doi.org/10.17081/just.26.39.4723

* Este producto corresponde a la fase de contextualización y caracterización realizada en el marco del Programa de Reconstrucción del Tejido social en zonas de Posconflicto en Colombia, código SIGP 57729. Proyecto: "Hilando capacidades políticas para las transiciones en los territorios"; financiado por el Fondo Nacional de Financiamiento para la Ciencia, la Tecnología y la Innovación, Francisco José de Caldas, con contrato No 213-2018 y Código 58960".

\title{
I. Introducción
}

En muchas ocasiones cuando se habla de paz se relaciona la palabra con violencia y guerra. La paz como disciplina de estudio se ha relacionado, en algunos países europeos y Estados Unidos, con las dos guerras mundiales del siglo XX y con los conflictos armados. A partir de ello, comienzan diferentes aproximaciones teóricas y reflexiones conceptuales sobre la guerra, y como lograr que esta no se repita de nuevo. Para los años sesenta, con el surgimiento de la investigación para la paz como una disciplina, se dan las primeras aproximaciones las cuales, se orientaban a determinar cómo prevenir la guerra. Algunos investigadores, activistas y académicos lograron ampliar el concepto de paz a partir de la comprensión de los diversos contextos conflictivos relacionándola con la justicia, la construcción social, la igualdad y el dialogo; lo que permitió avanzar hacia visiones abiertas y encontrar diferentes definiciones teniendo en cuenta a los actores involucrados, sus intereses políticos y sus entornos; partiendo de que la paz se ha convertido en una aspiración para la humanidad en el acontecer de la historia (López, 2011).

En esa línea de estudios, Calderón (2009) hace una revisión de la teoría de paz de Johan Galtung, el cual, es el primer autor que se aleja del paradigma occidental de pensar el hombre como bueno o malo y comienza a hacer referencia al sentido humano como una especie de categoría de mayor trascendencia en el análisis y un constructo que puede ser considerado patrimonio universal. Este autor logró concluir que, el ser humano es un ente con capacidad de paz, y que una constante en la historia de la humanidad es la trilogía: paz-violencia-humanidad en una lógica donde a mayor paz le correspondería menos violencia y viceversa. Surgen entonces alternativas al planteamiento: si quieres la paz, prepárate para la guerra, es decir, la idea de paz como resultado del sometimiento violento del hombre sobre el hombre; para dar paso 
a concepciones tales como: si quieres la paz, prepárate para la paz; lo que se convirtió en la gran tesis del citado autor: paz con medios pacíficos. Lo que se podría materializar, en palabras de (Pimienta et al., 2020), mediante la elaboración de acuerdos sociales incluyentes, la gestión pacifica de los conflictos, la adecuada relación con el Estado y la inclusión en Políticas Públicas con incidencia directa en el mejoramiento de las condiciones de vida.

Desde una perspectiva más general, se ve como la paz va tomando un nuevo rumbo y se empieza a perfilar un nuevo concepto como lo es, la paz imperfecta, representada por todas aquellas iniciativas en las que se consigue disminuir el conflicto, o, dicho de otra forma, se logra el máximo de paz posible, de acuerdo con las condiciones personales y sociales con que se inicia. Es decir, esta puede ser pensada como un proceso que está en construcción permanente, es dinámica y flexible. Además, cabe resaltar que la paz ha sido vista de diversas maneras y que estas corresponden al hecho histórico del momento, el contexto social en el que se desenvuelva y los intereses de los actores involucrados en su desarrollo (Muñoz, 2004), citado en (Loaiza, 2015).

Cabe resaltar que, la transición a la paz es posible si se da un cambio estructural en las formas de poder que se dan en el contexto de las regiones o zonas geográficas afectadas de alguna manera por el conflicto. Esta transición se logra, a través de la transformación social que bien podría iniciar con la reincorporación a la vida civil de los excombatientes, el cierre de las brechas socioeconómicas entre lo rural y lo urbano, la atenuación de las condiciones que sirvieron para la permanencia del conflicto armado, el fortalecimiento de la institucionalidad estatal, donde se mejoren las condiciones de seguridad y se construyan mejores oportunidades para todos, de tal forma que se pueda generar confianza en las comunidades locales (Martínez, 2019).

Dentro de este orden de ideas, Bautista (2017) plantea que, la paz territorial es un proceso de transformación con justicia social, que inicia con el reconocimiento de las vivencias pasadas de los sujetos y de las características específicas de los conflictos que vive en el presente; mediados por iniciativas de organización políticas, económica, social, cultural y ambiental, de las comunidades; lo que a su vez contempla, la necesidad de comprender múltiples dimensiones y escalas para una acción, que permita el paso hacia el goce pleno de las territorialidades, teniendo en cuenta las formas particulares de sentir y apropiar el espacio donde conviven las personas de tal forma que se logre construir un presente y un futuro colectivo, con respeto a la vida y búsqueda de bienestar.

Finalmente es importante referenciar que, en el contexto de paz territorial, toma relevancia el concepto de iniciativas locales entendidas como todos aquellos programas, proyectos, estrategias y acciones; adelantados por la sociedad civil, de manera autónoma y/o complementaria de las acciones estatales que, se desarrollan con una agenda propia para abordar y buscar solución a problemáticas asociadas con conflictos violentos. Su propósito generalmente es, construir alternativas pacíficas, para desestimular el uso de mecanismos violentos como estrategia de solución de conflictos y, promover a mediano y largo plazo la consolidación de sociedades más pacíficas; a través de acciones orientadas a mitigar las causas estructurales y coyunturales de las acciones violentas (Observatorio de Construcción de Paz, 2011). 


\section{Las Iniciativas locales como mediación para la paz}

Según García (2006) las iniciativas locales de paz son aquellas acciones adelantadas por una colectividad, ya sea una organización social debidamente establecida, formalizada y visible o solamente un grupo de personas, miembros de la comunidad, que se organiza con la finalidad puntual de rechazar las expresiones de violencia y/o tomar conciencia, discutir, visualizar, demandar o construir una alternativa de paz para la sociedad. No se excluyen las iniciativas estatales, siempre y cuando impliquen la participación activa de grupos sociales, diferentes a los funcionarios públicos. (p. 11).

En ese orden de ideas, González, Colmenares, \& Ramírez (2011), plantean que, las iniciativas locales están conformadas por pequeñas organizaciones que, han creado sus propios espacios donde actúan como defensoras de los derechos humanos, la creación de políticas públicas que beneficien a las comunidades, la protección de las comunidades víctimas, buscando su reconocimiento ante la institucionalidad como sujetos de derechos colectivos, impulsan la economía local por medio de productos tradicionales del municipio, realizan acciones concretas que, en pequeña escala son esfuerzos tangibles de resistencia social.

En el mismo sentido Mahecha, (2018) plantea que, las iniciativas locales abarcan diversos actores, pero sus protagonistas principales son organizaciones comunitarias con una base o estructura establecida en relación a la vida en comunidad, donde se planean y formulan proyectos sociales, culturales, productivos y ambientales; se da el reconocimiento y la observación de las relaciones con los diferentes actores armados que están en el territorio y además tienen una apuesta política autónoma y diferenciada que las hace ajenas a las prácticas políticas tradicionales de los municipios. Estas iniciativas tienen una agenda propia que les permiten gestionar recursos para su desarrollo, algunas han logrado tener acuerdos y ejecutar recursos de cooperación internacional, sin la ayuda de la institucionalidad local, solo con las capacidades que han desarrollado con la ejecución de las actividades en las comunidades. Además, han logrado consolidar procesos que construyen paz desde lo territorial que, al ser estables en el tiempo, se convierten en ejemplos para que no se repitan situaciones de conflicto una vez se da la transición del conflicto a la paz.

En lo esencial, estas experiencias se pueden definir como iniciativas de paz desde la base, reconociendo que tienen su origen en comunidades que vivieron de manera directa el impacto de la violencia, el conflicto armado o la corrupción administrativa. Las mismas presentan tres características importantes: la primera es que, son escenarios de construcción de paz que se materializan por medio de realidades vividas a través de procesos construidos por comunidades y/o pueblos completos, o por sectores poblacionales al interior de estas comunidades o pueblos, tales como mujeres, jóvenes o redes de organizaciones que fomentan iniciativas de paz, que tienen vida propia, aunque, en algunos casos, sean desconocidas; la segunda es que, contribuyen a profundizar la democracia en escenarios locales a través de la implementación de procesos de decisión participativos; y, la tercera es que, representan importantes alternativas para la paz, en la medida que buscan mejorar las condiciones de vida, teniendo en cuanta las capacidades y riquezas culturales de las comunidades, y mediante mecanismos no violentos como los diálogos humanitarios, la participación ciudadana, la resistencia civil, las manifestaciones culturales y los procesos organizativos, soportadas en sus propias necesidades (Hernández, 2002.) (Hernández 2008). 


\section{Orígenes del conflicto en el municipio de Ovejas - Departamento de Sucre}

El factor desencadenante del conflicto armado en la región de los Montes de María ha sido la lucha por la tierra, de manera particular en el municipio de Ovejas1, tiene que ver con la economía de enclave tabacalero que se soportó en una estructura productiva lesiva para el campesino; lo anterior, dio origen a las denominadas ligas campesinas que representaron las primeras agremiaciones de campesinos que surgieron con el objeto de disputar las tierras a los terratenientes. Dichas agremiaciones mutaron en los sindicatos tabacaleros, los cuales, lograron materializar las primeras recuperaciones de tierras mediante la parcelación de la finca la Europa por parte del instituto de fomento tabacalero. Este proceso del campesinado generó una respuesta violenta de los terratenientes, que se materializó en abusos de poder y amenazas y asesinatos de líderes campesinos. El rol preponderante del campesinado del municipio de Ovejas en la consolidación de la ANUC2, le significó a la comunidad amenazas, asesinatos y desapariciones; además de la estigmatización de las comunidades rurales como supuestas colaboradoras de organizaciones guerrilleras que surgieron o se asentaron en este municipio (Méndez, 2019).

\section{Experiencias de paz en el municipio de Ovejas - Departamento de Sucre}

En Colombia a partir del año 1988, durante la administración del gobierno del presidente Virgilio Barco y posteriormente entre los años 1990 a 1994 en el gobierno del presidente Cesar Gaviria, se implementó una política de paz negociada entre el gobierno y actores armados. Esta política permitió procesos de negociación con los movimientos armados: Partido Revolucionario de los Trabajadores (PRT), M-19, el Quintín Lame y el Ejército Popular de Liberación (EPL) (Centro de Memoria Paz y Reconciliación, 2012). Posteriormente, después de varios intentos fallidos en el año 2016, se logró un acuerdo de paz negociado con las guerrillas de las FERC-EP. El enfoque de paz negociada, puso en el debate la categoría de paz territorial, entendida, como la estrategia que vincula a los diversos actores del territorio en la construcción de paz desde las regiones (Daniels, 2015). La construcción de paz en Colombia se ha convertido entonces en un desafío para los territorios (Parra, 2014) y, como un mecanismo de defensa de los derechos básicos de las comunidades tales como la vida, la dignidad, el territorio, la autonomía, la integridad y la paz. (Mahecha, 2018).

De las iniciativas de paz negociadas en Colombia, el municipio de Ovejas ha sido escenario de diálogos, firmas de acuerdos e implementación de estrategias de paz en los siguientes procesos:

$\gg$ acuerdos entre el gobierno nacional y el Partido Revolucionario de los Trabajadores (PRT), los cuales se llevaron a cabo en el Corregimiento de Don Gabriel3 entre el 2 de agosto y el 28 de diciembre de 1990; el proceso concluyó con un acuerdo firmado el día 25 de enero de 1991 que, implicó la dejación de las armas, por parte del PRT y, la concesión, por parte del gobierno nacional, de una vocería permanente en la Asamblea Nacional Constituyente de 1991, las garantías y promoción para la transición del grupo guerrillero a la vida política y la formulación de un plan de reconciliación para la transición de los miembros del PRT a la vida civil, entre otros (Bejarano, Gonzalez, Jaramillo, Sierra, De Francisco, Roncallo, Hernandez, Álvaro; Gonzalez, Restrepo, Falla, Concha, 1991).

1 Municipio ubicado en la región de los Montes de María sucreño.

2 Asociación nacional de usuarios campesinos de Colombia-ANUC, La ANUC, es una asociación de tercer grado, de derecho privado, sin ánimo de lucro, con carácter de confederación a través de la cual se agremian los campesinos de Colombia (S.A.S., 2020).

3 Corregimiento del municipio de Ovejas Sucre. 
$\gg$ los diálogos y acuerdos con la denominada Corriente de Renovación Socialista - CRS4, los cuales, se desarrollaron entre miembros del gobierno nacional y representantes de este grupo insurgente, en el corregimiento Flor del Monte5, y que terminaron con la suscripción de un acuerdo sobre tres aspectos básicos: la reincorporación a la vida política y democrática de la Corriente como organización; el desarrollo social con la comunidad y los territorios donde la CRS tuvo presencia armada; y los beneficios económicos, sociales y jurídicos, de reinserción para los miembros del grupo insurgente. Producto de este proceso, se desarrolló un programa de dotación de tierras implementado por el INCORA para mejorar las condiciones de las comunidades campesinas en Ovejas (Rettberg, 2006).

$\gg$ Finalmente, en el año 20016, en la Habana-Cuba, se firmó el acuerdo de paz entre las FARC-EP y el gobierno nacional, el cual, se estructuró sobre seis puntos o ejes transversales: 1) Reforma rural integral, que busca sentar las bases para la transformación del campo, reversando los efectos del conflicto y las condiciones que han facilitado la persistencia de la violencia en el país. 2) Participación política, donde se busca ampliar y cualificar la democracia como condición para lograr bases sólidas para forjar la paz. 3) Fin del Conflicto, donde se establecen los términos para finalizar el conflicto armado. 4) el problema de las drogas ilícitas orientado a encontrar una solución definitiva al problema de las drogas ilícitas. 5) Victimas Este acuerdo busca -a través de los cinco mecanismos y medidas que integran el Sistema Integral de Verdad, Justicia, Reparación y No Repetición- lograr la satisfacción de los derechos de las víctimas, asegurar la rendición de cuentas por lo ocurrido, garantizar la seguridad jurídica de quienes participen en él, y contribuir a garantizar la convivencia, la reconciliación y la no repetición, como elementos esenciales de la transición a la paz. 6) Implementación, verificación y refrendación el cual, señala que con la firma del Acuerdo Final y su refrendación ciudadana, se da inicio a la implementación de todos los puntos acordados (Publicación De, n.d.). La implementación de la Reforma Rural Integral, se focalizó a través de los planes de ordenamiento con enfoque territorial -PEDET, los cuales se realizarán en 16 subregiones y 170 municipios. Dentro de las 16 subregiones, se ubica los Montes de María, que abarca municipio del departamento de Bolívar y Sucre, dentro de los municipios del departamento de Sucre, entre otros, se contempla el municipio de Ovejas (DNP, 2018).

Los referentes analizados, dan cuenta de que el municipio de Ovejas, alberga una comunidad que ha estado en medio del conflicto armado y de igual forma ha sido escenario de reconciliación y procesos de paz. En tal sentido, se hace necesario comprender la incidencia que ha tenido la sociedad civil en los procesos de construcción de paz, en los territorios; analizando para ello, las iniciativas locales que han contribuido en la búsqueda del camino hacia la paz, las cuales, se han convertido en un elemento de mediación relevante para el fortalecimiento del tejido social y la identificación de capacidades que han quedado instituidas por la salida negociada con algunos actores del conflicto armado en Colombia.

$\begin{array}{ll}4 & \text { Grupo insurgente que se reconoce como una disidencia del ELN. } \\ 5 & \text { Corregimiento del municipio de Ovejas, Sucre. }\end{array}$ 
Teniendo en cuenta lo anterior, es pertinente preguntarse: ¿Cuáles son las iniciativas locales que han actuado como herramientas de mediación para la construcción de paz en el municipio de Ovejas, Departamento de Sucre?

El presente artículo analiza las iniciativas locales que actúan como herramientas de mediación para la construcción de paz en el municipio de Ovejas, que si bien son diversas, corresponden a las visiones de paz negociada que poseen las comunidades, teniendo en cuenta los contextos donde se desenvuelven, con lo cual, aportan a la reconstrucción del territorio, generando lenguajes transformadores alrededor de la paz y procesos de formación política e identidades colectivas alrededor de un objetivo común.

\section{Método}

Se desarrolló un estudio con enfoque cualitativo y diseño descriptivo transversal. Se utilizaron, como técnica de recolección de datos, la revisión documental y la entrevista abierta. La época de recolección de información, fue el segundo semestre de 2019. El estudio tuvo como población objetivo, las organizaciones formales o, de hecho, que se han desarrollado en las zonas rural y urbana del Municipio de Ovejas, como iniciativas locales de paz. Para el análisis de la información se organizaron categorías teniendo en cuanta las tipologías de organizaciones campesinas, productivas, indígenas, de mujeres, de víctimas, y juntas de acción comunal, con el objeto de comprender su foco de actuación (Hernández Sampieri, Fernández Collado, \& Baptista Lucio, 2004) (Hurtado de Barrera, 2012).

\section{Resultados de investigación}

Según (Aguilera, 2013), entre los años 1999 a 2011, se han desarrollado varias acciones y estrategias, orientadas a coadyuvar la paz territorial en la región de los Montes de María, tal como se describe en le tabla 1. Entre estas, el Programa de Desarrollo y Paz de Montes de María (PDP) a través del Tercer Laboratorio de Paz, logró que las organizaciones sociales, comunitarias y populares, en la subregión, en términos generales, y en Ovejas, de manera particular, renacieran e impulsaran acciones con miras a la exigibilidad de los derechos de las comunidades, la defensa del territorio y la interlocución con las administraciones locales. Todo este ejercicio les ha facilitado la participación en el diseño de políticas públicas, para ello, participaron en la construcción del capítulo de victimas del Plan de Desarrollo, la política de atención a las víctimas, los procesos de reparación colectiva, la creación de una zona de reserva e instalación de la Mesa Regional Campesina y la restitución de tierras que se adelanta en el municipio, entre otros. (Méndez, 2017). 
TABLA 1. ACCIONES DE PAZ Y RECONSTRUCCIÓN DEL TEJIDO SOCIAL EN LA REGIÓN DE LOS MONTES DE MARÍA.

\begin{tabular}{|c|c|c|c|}
\hline Año & Proponentes & Acciones & Propósito \\
\hline 1999 & $\begin{array}{l}\text { Oficina del Comisionado de Paz, } \\
\text { Gobernación de los departamentos } \\
\text { de Sucre y Bolívar, Ministerio de } \\
\text { Educación Nacional-MEN. }\end{array}$ & $\begin{array}{l}\text { Se formuló el PDIMM (Plan para el } \\
\text { Desarrollo Integral de los Montes } \\
\text { de María) }\end{array}$ & $\begin{array}{l}\text { Construir un instrumento para orientar } \\
\text { las intervenciones institucionales en } \\
\text { la región. }\end{array}$ \\
\hline 2001 & $\begin{array}{l}\text { Gobiernos de los departamentos de } \\
\text { Sucre y Bolivar }\end{array}$ & $\begin{array}{l}\text { Se desarrolló un Convenio de } \\
\text { Competitividad Territorial para co- } \\
\text { adyuvar el Desarrollo Económico y } \\
\text { Social de la región. }\end{array}$ & $\begin{array}{l}\text { Coadyuvar la competitividad territorial, } \\
\text { a través de un Plan para el Desarrollo } \\
\text { Integral de los Montes de María. }\end{array}$ \\
\hline 2001 & Misión FAO y Ministerio de Agricultura & $\begin{array}{l}\text { Programa Especial de Seguridad } \\
\text { Alimentaria (PESA) }\end{array}$ & $\begin{array}{l}\text { Contribuir en el mejoramiento de las } \\
\text { condiciones de vida, de la población } \\
\text { rural de la región. }\end{array}$ \\
\hline 2002 & Gobierno nacional y Naciones Unidas & $\begin{array}{l}\text { Se decretó la región como Zona } \\
\text { de Rehabilitación y Conciliación } \\
\text { dentro del marco de la Política de } \\
\text { Seguridad Democrática. }\end{array}$ & $\begin{array}{l}\text { Poner en marcha programas de desar- } \\
\text { rollo económico, social y ambiental y } \\
\text { aumentar el pie de fuerza pública en } \\
\text { la región. }\end{array}$ \\
\hline 2003 & $\begin{array}{l}\text { Universidad de Cartagena, Programa } \\
\text { de Desarrollo de las Naciones Unidas, } \\
\text { Gobiernos departamentales de Sucre y } \\
\text { Bolívar, Corporación Territorios. }\end{array}$ & $\begin{array}{l}\text { Se creó el Programa de Desarrollo } \\
\text { y Paz de los Montes de María - } \\
\text { PROMONTES }\end{array}$ & $\begin{array}{l}\text { Mejorar infraestructura física, propiciar } \\
\text { el desarrollo humano, a través de la re- } \\
\text { construcción del tejido social, fortalecer } \\
\text { la gobernabilidad local y mejorar la } \\
\text { productividad como una estrategia para } \\
\text { garantizar la seguridad alimentaria en } \\
\text { la región. }\end{array}$ \\
\hline 2004 & $\begin{array}{l}\text { Arquidiócesis de Cartagena, Sincelejo, } \\
\text { y Magangué y Justa paz }\end{array}$ & $\begin{array}{l}\text { Se creó la Fundación Red de } \\
\text { Desarrollo y Paz de los Montes } \\
\text { de María }\end{array}$ & Desarrollar las actividades PROMONTES. \\
\hline 2004 & $\begin{array}{l}\text { Convenio Gobierno Nacional y la } \\
\text { Comisión Europea }\end{array}$ & $\begin{array}{l}\text { Se inició el Tercer Laboratorio } \\
\text { de Paz y se creó el Observatorio } \\
\text { Regional de Cultura, Política, Paz, } \\
\text { Convivencia y Desarrollo de los } \\
\text { Montes de María. }\end{array}$ & $\begin{array}{l}\text { Construir activos sociales, desarrol- } \\
\text { lar activos económicos; y mejorar la } \\
\text { gobernabilidad. }\end{array}$ \\
\hline 2004 & Gobierno Nacional & $\begin{array}{l}\text { Se creó un Centro de Coordinación } \\
\text { de Acción Integral - CCAl, integra- } \\
\text { do por personal militar y civil. }\end{array}$ & $\begin{array}{l}\text { Hacer una integración entre estrategias } \\
\text { militares, económicas y sociales, de tal } \\
\text { forma que la fuerza pública pudiera } \\
\text { tener control sobre la región y al } \\
\text { mismo tiempo se pudieran implementar } \\
\text { programas de generación de ingresos y } \\
\text { reconstrucción del tejido social. }\end{array}$ \\
\hline 2008 & $\begin{array}{l}\text { Ministerios del Interior y de Justicia, de } \\
\text { Protección Social, de Educación, y de } \\
\text { Agricultura, el ICFES, Incoder, el SENA, } \\
\text { Coldeportes, la Agencia Presidencial } \\
\text { para la Acción Social y la Cooperación } \\
\text { Internacional, las Gobernaciones } \\
\text { y Alcaldías, las Fuerzas Militares y } \\
\text { de Policía, la Fiscalía General de la } \\
\text { Nación y la Registraduría Nacional del } \\
\text { Estado Civil. }\end{array}$ & $\begin{array}{l}\text { Se creó el Centro de Coordinación } \\
\text { Regional de los Montes de María } \\
\text { - CCR }\end{array}$ & $\begin{array}{l}\text { Implementar la estrategia del (CCAI) e } \\
\text { iniciar procesos de titulación de tierras. }\end{array}$ \\
\hline 2011 & INCODER & Zonas de reserva campesina & $\begin{array}{l}\text { Propiciar el retorno de comunidades de } \\
\text { la región que fueron desplazadas en la } \\
\text { época del conflicto armado, implemen- } \\
\text { tar programas de asistencia técnica, } \\
\text { educación e infraestructura, con miras a } \\
\text { convertir, al pequeño productor campes- } \\
\text { ino, en empresario del campo. }\end{array}$ \\
\hline 2011 & 156 Organizaciones de base & $\begin{array}{l}\text { Mesa Regional Campesina de los } \\
\text { Montes de María }\end{array}$ & $\begin{array}{l}\text { Fomentar la participación de las comu- } \\
\text { nidades campesinas en las decisiones } \\
\text { de políticas públicas y promover } \\
\text { procesos organizativos para coadyuvar } \\
\text { el empoderamiento político social, , } \\
\text { cultural, económico y ambiental. }\end{array}$ \\
\hline
\end{tabular}

Fuente: elaboración propia, con basa a (Aguilera, 2013). 
En términos puntuales, se encontraron en Ovejas, alrededor de 60 iniciativas locales que están representadas en organizaciones sociales, productivas, comunitarias y de víctimas, como se presenta en la tabla 2, que se relacionan con los gobiernos nacional y municipal y la cooperación internacional, con la finalidad de desarrollar procesos o acciones que permitan la reconstrucción del tejido social, la gobernabilidad participativa, la promoción y desarrollo de actividades académicas, todo con miras a buscar el bien común y dar respuesta a las problemáticas del municipio.

\section{TABLA 2. INICIATIVAS LOCALES PARA CONSTRUCCIÓN DE PAZ TERRITORIAL EN EL MUNICIPIO DE OVEJAS - SUCRE.}

\begin{tabular}{|c|c|c|}
\hline Tipo De Organización & Organizaciones & Enfoque \\
\hline $\begin{array}{l}\text { Organizaciones Cam- } \\
\text { pesinas }\end{array}$ & $\begin{array}{l}\text { Anuc Ovejas, } \\
\text { Asociación de campesinas y campesinos de la Europa } \\
\text { Asociación de retornados de Ovejas - ASOCARES. } \\
\text { Asociación de Campesinos y Campesinas Baluarte } \\
\text { Asociación Campesina Victimas Retornadas de la Vereda Borrachera } \\
\text { Asociación de usuarios campesinos de Canutalito } \\
\text { Asociación de campesinos de Agua Rica } \\
\text { Asociación de Campesinos y Retornados de Don Gabriel } \\
\text { Asociación de campesinos de la Cantaleta } \\
\text { Asociación de Campesinos Desplazados de El Zapato } \\
\text { Asociación de usuarios campesinos corregimiento de San Rafael }\end{array}$ & $\begin{array}{l}\text { Trabajan por la soberanía y seguridad alimen- } \\
\text { taria, la comercialización de los productos } \\
\text { tradicionales de la región, la lucha por la } \\
\text { recuperación de la tierra y el uso adecuado } \\
\text { del suelo }\end{array}$ \\
\hline $\begin{array}{l}\text { Representatividad } \\
\text { indigena }\end{array}$ & $\begin{array}{l}\text { Parcialidad San José de Almagra, } \\
\text { Parcialidad de Galapa } \\
\text { Parcialidad de Vilu }\end{array}$ & $\begin{array}{l}\text { Trabajan por organizar la parcialidad en temas } \\
\text { relacionados con defender los derechos de } \\
\text { la comunidad indígena de la zona, además se } \\
\text { dedican al campo en actividades de agricultu- } \\
\text { ra y pecuarias }\end{array}$ \\
\hline $\begin{array}{l}\text { Organizaciones pro- } \\
\text { ductivas }\end{array}$ & $\begin{array}{l}\text { Asociación del Distrito de riego de tierras el Flechal, } \\
\text { Asociación De Tabacaleros De Ovejas (ASOTAOVEJAS), } \\
\text { Empresa Comunitaria San Rafael, } \\
\text { Asociación de Productores Agrícolas Retornados Canutal, } \\
\text { Asociación Agropecuaria de víctimas de la Peña } \\
\text { Asociación municipal de productores agrícola de Ovejas. } \\
\text { Asociación de Agricultores Victimas del Conflicto de la Vereda Alemania } \\
\text { Asociación de emprendedores agropecuarios Los Limos (LOSPINTAMONOS) } \\
\text { Asociación de vivienda de Almagra } \\
\text { Asociación de empresarios rurales de Canutalito } \\
\text { Asociación de productores del Tesoro } \\
\text { Asociación de pequeños productores agropecuarios victimas retornados de Don } \\
\text { Gabriel } \\
\text { Asociación de agricultores El Palmar - ASOGRIP } \\
\text { Asociación de Agricultores de Flor del Monte - ASOAGRIFLOR } \\
\text { Asociación de Productores Agropecuarios de Las Babillas (APROBA) } \\
\text { Asociacion de retornados Nuevo Porvenir - San Francisco } \\
\text { Precooperativa Agropecuaria de Reubicados de Salitral } \\
\text { Asociación De Cultivadores Desplazados y Retornados Del Corregimiento de Salitral } \\
\text { Asociación municipal de productores agrícola de Ovejas } \\
\text { Asociación De Productores De San Rafael - ASOPROSAL } \\
\text { Asociación Agrícola de Desplazados de San Rafael (AGRODESAR) } \\
\text { Asociación de Pequeños productores de Santa Fé }\end{array}$ & $\begin{array}{l}\text { Se dedican a la producción agropecuaria con } \\
\text { énfasis en lo agrícola y pecuario, se resalta } \\
\text { que el } 70 \% \text { de estas organizaciones están } \\
\text { representadas legalmente por mujeres. }\end{array}$ \\
\hline $\begin{array}{l}\text { Organizaciones de } \\
\text { mujeres }\end{array}$ & $\begin{array}{l}\text { Corporación Narrar para Vivir } \\
\text { Asociación de mujeres de San Francisco } \\
\text { Asociación de mujeres víctimas de San Rafael. } \\
\text { Asociación de mujeres campesinas negras e indigenas y desplazadas del corregimien- } \\
\text { to Canutal } \\
\text { Asociación de mujeres Incluyentes del Corregimiento de San Rafael - AMINSA } \\
\text { Asociación de productores Agropecuarios de Villa del Carmen - ASOPROVICA }\end{array}$ & $\begin{array}{l}\text { Trabajan sobre la prevención de la violencia } \\
\text { contra la mujeres, proyectos productivos y } \\
\text { acceso a la justica de género; la identidad } \\
\text { de la mujer como sujeto de derecho y en el } \\
\text { fortalecimiento en el empoderamiento social } \\
\text { y político. }\end{array}$ \\
\hline $\begin{array}{l}\text { Organizaciones de } \\
\text { víctimas }\end{array}$ & $\begin{array}{l}\text { Asociación de Víctimas de Chengue (ASOVICHENGUE), } \\
\text { Asociación de Derechos de las Victimas de Canutalito, } \\
\text { Ovejas diversa } \\
\text { Asociación de Desplazados de Almagra. } \\
\text { Asociación de Retornados Bajo La Palma } \\
\text { Asociacion de desplazados de Canutal } \\
\text { Asociación de desplazados de la Chavela (AsChavela) } \\
\text { Comite de impulso } 6 \text { veredas }\end{array}$ & $\begin{array}{l}\text { Trabajan en la Mesa Municipal de Victimas ya } \\
\text { que son representantes de la misma, además } \\
\text { participaron en la construcción de la política } \\
\text { pública de víctimas del municipio para el plan } \\
\text { de desarrollo } 2016 \text { - } 2019\end{array}$ \\
\hline $\begin{array}{l}\text { Juntas de acción } \\
\text { comunal }\end{array}$ & $\begin{array}{l}\text { JAL El Palmar, } \\
\text { JAL La Peña, } \\
\text { JAL San Francisco. } \\
\text { JAL Vereda Agua Rica } \\
\text { JAL Vereda El Flechal } \\
\text { JAL Corregimiento San Rafael } \\
\text { JAL De Almagra }\end{array}$ & $\begin{array}{l}\text { Trabajan en los procesos de reparación } \\
\text { colectiva de los corregimientos y veredas. } \\
\text { Además son las que ayudan a solucionar los } \\
\text { problemas de la comunidad y permiten la } \\
\text { organización de actividades. }\end{array}$ \\
\hline
\end{tabular}

Fuente: elaboración propia. 
Estas iniciativas locales han realizado acciones encaminadas a la construcción de paz en el municipio de Ovejas, donde se muestra el aporte que estas hacen continuamente para buscar el bien común de las comunidades a las cuales representan. Entre otras, se resaltan las siguientes actividades: a) Participación en la construcción del Plan de Desarrollo con Enfoque Territorial - PDET; b) Gestión y participación activa en los procesos de reparación colectiva con la Unidad para las Victimas. Actualmente se adelantan 5 procesos de reparación colectiva (6 Veredas (Villa Colombia, Medellín, El Palmar, San Francisco, La Coquera y Borrachera), Flor del Monte y La Peña, Chengue, San Rafael y El Tesoro). C) Construcción de la política pública de Victimas en el Plan de Desarrollo 2016 - 2019. d)Trabajos sobre recuperación de la memoria histórica de las zonas rurales. e) Impulso de la Zona de Reserva Campesina en Montes de María por medio de las organizaciones campesinas. f) Defensa del territorio y los recursos naturales (agua, bosque), ante la presencia de exploración/explotación de gas y petróleo en el territorio ovejero.g) Impulso de la economía local desde la producción y comercialización de los productos de la zona. h) La lucha por la recuperación de las tierras despojadas y abandonadas por las lógicas del conflicto armado. i) La reintegración de excombatientes de las FARC a los territorios.

\section{Discusiones}

Las iniciativas locales que, se desarrollan en el municipio de ovejas, presentadas en la tabla 2, dan cuenta de la gestión de paz con enfoque territorial que, ha venido tomando fuerza en Colombia, teniendo en cuenta que, estas iniciativas, abarcan diversos actores, pero sus protagonistas principales son organizaciones comunitarias que formula e implementan proyectos sociales, culturales, productivos y ambientales; como lo plantean Parra (2014), Daniels (2015) y Mahecha (2018).

Los hallazgos encontrados con respecto al número y tipo de iniciativas locales vigentes en el municipio de Ovejas, evidencian que dichas iniciativas, están constituidas por pequeñas organizaciones, que, han creado sus propios espacios, son desarrolladas por un colectivo social, impulsan la economía local y la gestión de políticas públicas para beneficio de las poblaciones, como lo plantea García (2006) y Gonzales, Colmenares \& Ramirez (2011).

En lo que se refiere a los resultados de las iniciativas locales, los hallazgos, son coherentes con lo planteado por López (2011), en el sentido de que las acciones que se evidencian en las comunidades del municipio de Ovejas, reflejan el hecho de que la paz se convirtió en un deseo de la humanidad, se observa además, el enfoque planteado por Johan Galtun, citado en Caldero (2009), teniendo en cuanta que, se percibe una visión de paz enmarcada en el enfoque, si quieres la paz prepárate para la paz, es decir, la paz construida con medios pacíficos y se observa además lo planteado por Pimienta et al.,(2020) con respecto a la gestión pacífica del conflicto a través de la inclusión en políticas públicas con incidencia directa en el mejoramiento de la calidad de vida de las comunidades.

Finalmente es importante resaltar, que en la tabla 1, se referencia la estrategia del Centro de Coordinación de Acción Integral, la cual implicaba una estructura organizativa entre autoridades militares y civiles, para coadyuvar la titulación de tierras, la reactivación económica, la reconstrucción del tejido social, el fortalecimiento de la gobernabilidad, y la construcción de infraestructura y, la cual, es una estrategia implementada a partir del año 2004, donde estaba en auge la política de seguridad democrática del gobierno del presidente Álvaro Uribe (Aguilera, 2013). Se percibe que, esta estrategia estaba enmarcada en el paradigma si quieres la paz, prepárate para la guerra, referenciado en Calderón (2009), donde se piensa, que la paz se logra producto de la derrota militar de uno de los actores armados sobre el otro u otros. 


\section{Conclusiones}

Las iniciativas locales desarrolladas en el municipio de Ovejas, Sucre, como herramientas de paz; dan cuenta del enfoque territorial, en procesos de construcción de paz, las cuales, están representadas por al alrededor de 60 iniciativas que, se pueden categorizar dentro de las tipologías de organizaciones campesinas, productivas, de mujeres, de víctimas y parcialidades indígenas; las cuales, han participado de la construcción del PDET, la gestión de reparación colectiva de víctimas del conflicto armado, construcción de políticas públicas, recuperación de la memoria histórica, impulso a la zona de reserva campesina, defensa del territorio y los recursos naturales e impulso a la economía local. Estas iniciativas son producto de estrategias nacionales y regionales que, con apoyo de organismos internacionales, se han implementado en la región de los Montes de María, y en el estudio de las mismas se logra evidenciar, la polarización que se vive a nivel nacional sobre cuál es el mejor camino para la construcción de paz: el enfoque, si quieres la paz, prepárate para la guerra, o el enfoque, si quieres la paz, prepárate para la paz.

\section{Referencias Bibliográficas}

Aguilera, M. (2013). Montes de María : una subregión de economía campesina y empresarial. Documentos de Trabajo Sobre Economía Regional y Urbana; No. 195, 1-93. http://repositorio.banrep.gov.co/ handle/20.500.12134/3033

Bautista, S. (2017). Contribuciones a la fundamentación conceptual de paz territorial. Revista Ciudad Pazando, Vol. 10 No. 1, PP 100-110. Recuperado de: https://doi.org/10.14483/2422278X.11639

Bejarano, Jesus Antonio; Gonzalez, Valentín; Jaramillo, Carlos Eduardo; Sierra Sergio; De Francisco, Gonzalo; Roncallo , Pablo; Hernandez, Álvaro; Gonzalez, Rafael; Restrepo, Gabriel; Falla, Ernesto; Concha, S. T. (1991). Acuerdo final PAZ PRT, en Don Gabriel, municipio de Ovejas, Sucre.

Calderón Concha, P. (2009). Teoría de confl ictos de Johan Galtung Th eory of Confl icts by Johan Galtung.

Centro de Memoria Paz y Reconciliación. (2012). Los procesos de paz con el M-19, el EPL, el Quintin Lame y PRT desde 1989 a 1991. 1-6. http://www.indepaz.org.co/wp-content/uploads/2013/04/Los_proceso_ de_paz_con_el_M19-EPL-Quintín-Lame-1989-1991.pdf

DNP. (2018). Plan Marco de Implementación del Acuerdo Final para la Terminación del Conflicto y la Construcción De una Paz estable y duradera. https://colaboracion.dnp.gov.co/CDT/Conpes/ Económicos/3932_Anexo B_Plan Marco de Implementación (PMI).pdf

García-Durán, M. (2006). Paz en el territorio: dinámica de expansión geográfica del accionar colectivo por la paz en Colombia 1978-2003. Territorios, núm. 15, pp. 9-32. Recuperado de http://www.redalyc.org/ articulo.oa?id=35711624002

González, S; Colmenares, J; Ramírez, V. (2011). La resistencia social: una resistencia para la paz. Hallazgos, vol. 8, núm. 15, enero-junio, 2011, pp. 237-254. Recuperado de http://www.redalyc.org/articulo. oa?id $=413835204013$

Hernández, E. (2002). La Paz y la No violencia adquieren significado propio en Colombia en las iniciativas de Paz que construyen las bases desde lo local. Convergencia. Revista de Ciencias Sociales, vol. 9, núm. 30. Recuperado de http://www.redalyc.org/articulo.oa?id=10503009

Hernández, E. (2008a). La Paz imaginada por quienes la construyen: Iniciativas Civiles de Paz de Base Social identifican sus sueños de Paz. Reflexión Política, vol. 10, núm. 19, pp. 134-147. Recuperado de https:// www.redalyc.org/articulo.oa?id=11001911 
Hernández Sampieri, R., Fernández Collado, C., \& Baptista Lucio, P. (2004). Metodología de La Investigación. Mexico: Mc Graw Hill.

Hurtado de Barrera, J. (2012). Metodología de la Investigación "guía para la comprensión holística de la ciencia". Bogotá-Caracas: Quiron.

Loaiza, A.M. (2015). Investigación para la paz y trabajo social: construcción de una cultura para las paces con perspectiva de género. Revista Eleuthera, 12, 89-111. DOI: 10.17151/eleu.2015.12.5.

López, M. (2011). Reflexiones sobre las desigualdades en el contexto de los estudios de paz. Revista Paz y Conflicto, 4, 121-135. ISSN: 1988-7221.

Mahecha, J. (2018). Iniciativas locales de paz: tres casos desde la resistencia civil para la reflexión. Revista Ciencia Política, Vol 13 No. 26, PP. 153-181. Recuperado de: https://www.academia.edu/39005768/ Iniciativas_locales_de_paz_Tres_casos_desde_la_resistencia_civil_para_la_reflexión

Martínez, H. (2019). Insistamos en la paz territorial. Dejusticia. Recuperado de: https://www.dejusticia.org/ column/insistamos-en-la-paz-territorial/

Méndez, F. (2017). El conflicto armado y la violencia en Ovejas 1997-2007: “Memorias de la guerra”. Tesis. Universidad de Cartagena

Observatorio de Construcción de Paz. (2011). Estado y ciudadanía para la Paz. Obtenido detitan.utadeo.edu. co:http://titan.utadeo.edu.co/comunidad/paz/images/stories/documentos/Serie1.pdf

Parra, L. (2014). Prácticas y experiencias colectivas ante la guerra y para la construcción de paz: Iniciativas Sociales de Paz en Colombia. Revista Reparación a las victimas de dictaduras, conflictos armados y violencia política. V. 14. No 2. PP. 311- 703. Recuperado de: http://www.scielo.org.co/pdf/agor/v14n2/ v14n2a02.pdf

Pimienta, J. L. R., Gulfo, Y. E. C., \& Sagbini, A. J. D. (2020). Estrategias de ciudadanía juvenil aplicadas en la formación de agentes de Paz y Derechos Humanos. Justicia, 25(38), 1-12. https://doi.org/10.17081/ just.25.38.4455

Publicación De, U. (n.d.). El Acuerdo Final de paz la oportunidad para construir paz. Retrieved October 31, 2020, from www.mesadeconversaciones.com.co

Rettberg, A. (2006). Colección INFORMES ESPECIALES. http://www.pnud.org.co

S.A.S., A. (2020). Quieénes Somos. 1-3. http://www.anuc.co/quienes-somos.asp 